\title{
Organoboron Quinolinolates with Extended Conjugated Chromophores: Synthesis, Structure, Electronic and Electroluminescent Properties
}

\section{Supporting Material}

Stefan Kappaun, ${ }^{a}$ Stephan Rentenberger, ${ }^{b}$ Alexander Pogantsch, ${ }^{b}$ Egbert Zojer,${ }^{b}$ Kurt Mereiter,${ }^{c}$ Gregor Trimmel, ${ }^{a}$ Robert Saf, ${ }^{a}$ Kai C. Möller, ${ }^{d}$ Franz Stelzer, ${ }^{a}$ and Christian Slugovc ${ }^{* a}$

${ }^{a}$ Institute of Chemistry and Technology of Organic Materials (ICTOS), Graz University of Technology, Stremayrgasse 16, A-8010 Graz, Austria,

${ }^{\mathrm{b}}$ Institute of Solid State Physics, Graz University of Technology, Petersgasse 16, A-8010 Graz, Austria

${ }^{\mathrm{c}}$ Institute of Chemical Technologies and Analytics, Vienna University of Technology, Getreidemarkt 9/164, A-1060 Vienna, Austria, and

${ }^{\mathrm{d}}$ Institute for Chemical Technology of Inorganic Materials (ICTAS), Graz University of Technology, Stremayrgasse 16, A-8010 Graz, Austria

\section{CONTENT}

MALDI TOF MS data

page $2-5$

${ }^{1} \mathrm{H}$ and ${ }^{13} \mathrm{C}$ NMR spectra page 6-9

Photoluminescence of films made of $\mathbf{9 - 1 2}$ page 10

Photograph of a film of 9 page 10

Current density - voltage characteristics of devices made of $\mathbf{9 , 1 0}$ and $\mathbf{1 2}$ page 11-12 
9 (=SKA051) $\mathrm{C}_{21} \mathrm{H}_{14} \mathrm{BBr}_{2} \mathrm{NO} \quad \mathrm{M}_{\mathrm{av}}=466.9782$

m rel.int.

$463.9572 \quad 0.1160$

$464.9539 \quad 0.5035$

$465.9558 \quad 0.3452$

$466.9520 \quad 1.0000$

$467.9545 \quad 0.3436$

$468.9504 \quad 0.5107$

$469.9532 \quad 0.1156$

$470.9562 \quad 0.0140$

$471.9591 \quad 0.0012$
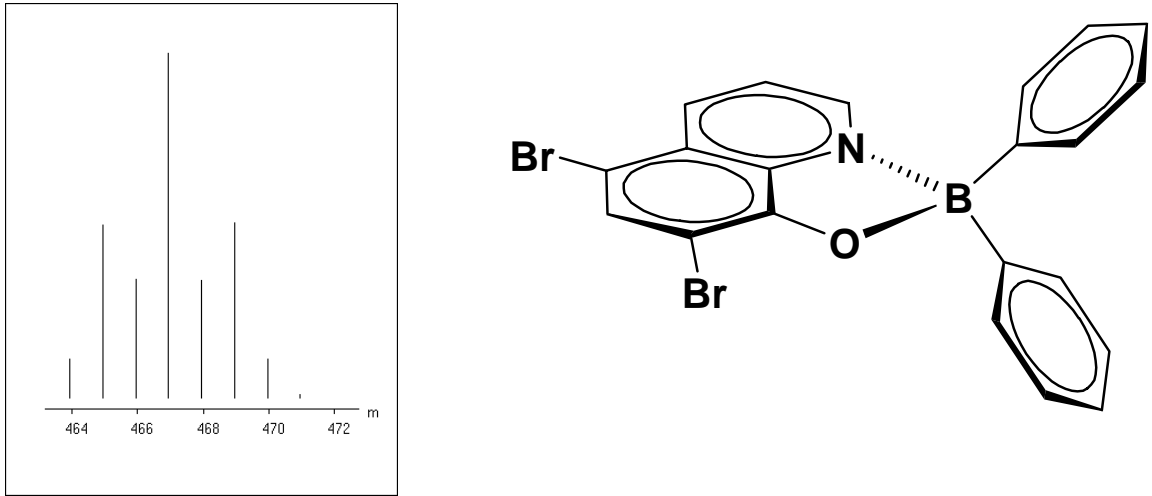

SKA051 (0.045) Is $(1.00,0.10) \mathrm{C} 15 \mathrm{H} 9 \mathrm{BBr} 2 \mathrm{NO}$
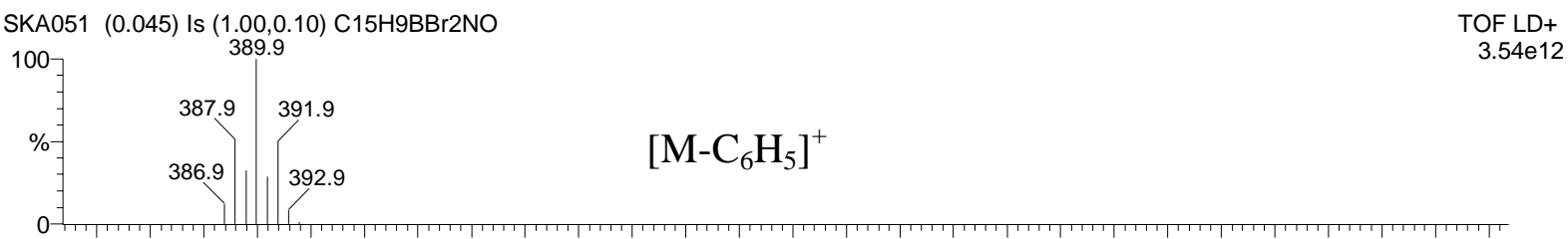

$3.54 \mathrm{e} 12$
3

$\left[\mathrm{M}-\mathrm{C}_{6} \mathrm{H}_{5}\right]^{+}$

SKA051 $(0.045)$ Is $(1.00,0.10)$ C21H14BBr2NOH
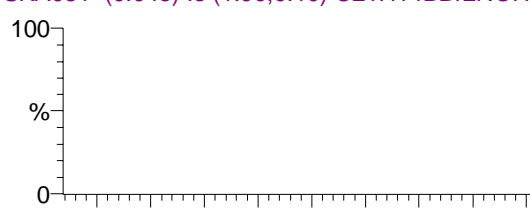

SKA051 (0.045) Is $(1.00,0.10) \mathrm{C} 21 \mathrm{H} 14 \mathrm{BBr} 2 \mathrm{NONa}$

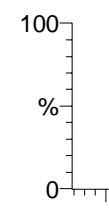

SKA051 55 (2.704) Cm (1:120)
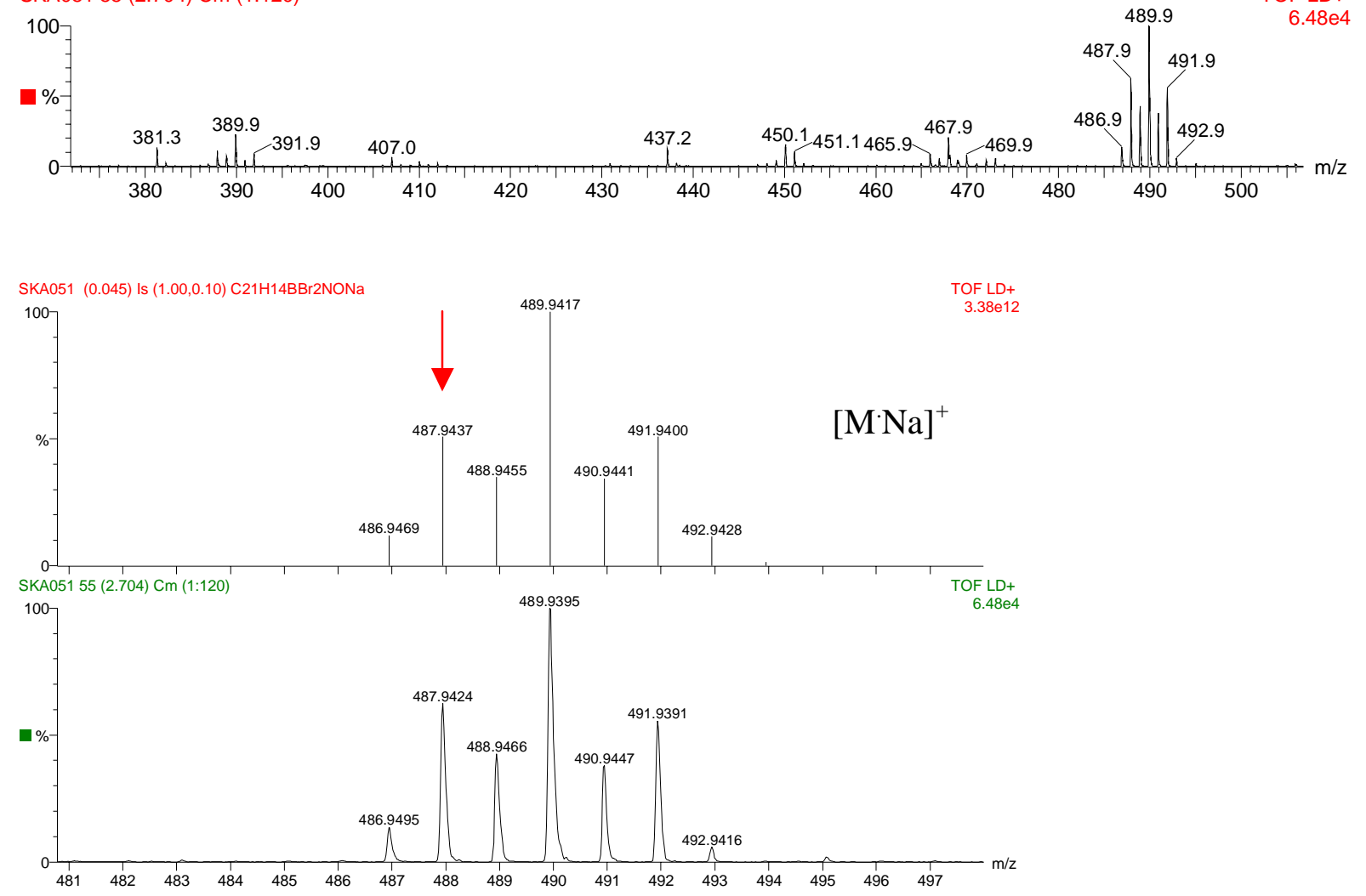
m rel.int.

$460.1987 \quad 0.2234$

$461.1957 \quad 1.0000$

$462.1987 \quad 0.3611$

$463.2019 \quad 0.0671$

$464.2050 \quad 0.0084$
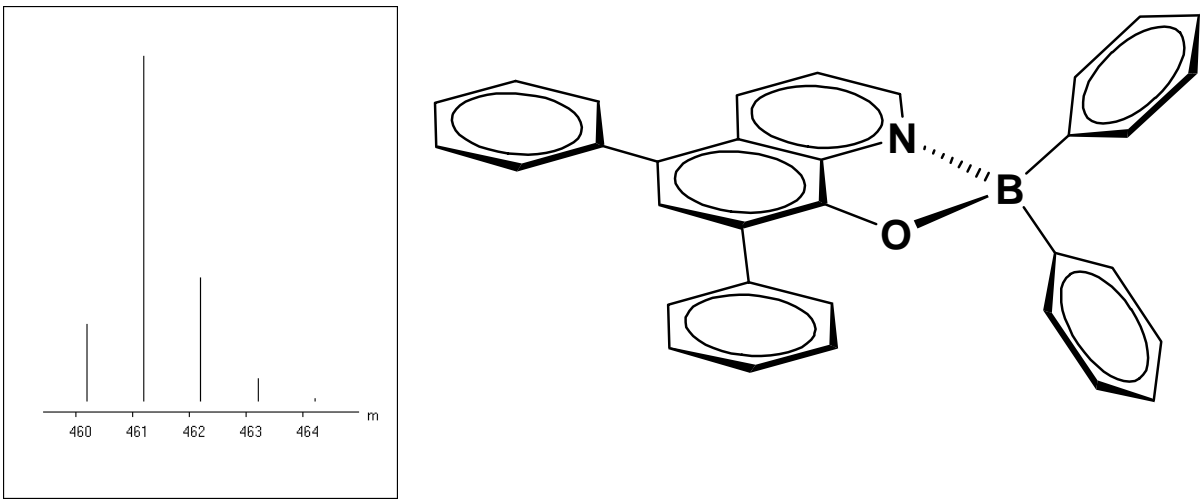

SKA058B $(0.051)$ Is $(1.00,0.10) \mathrm{C} 27 \mathrm{H} 19 \mathrm{BNO}$

TOF LD+

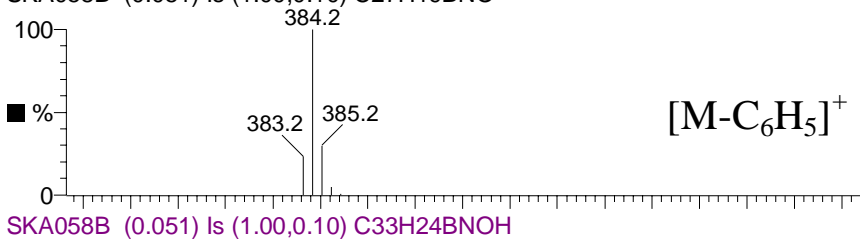

$6.34 \mathrm{e} 12$

100

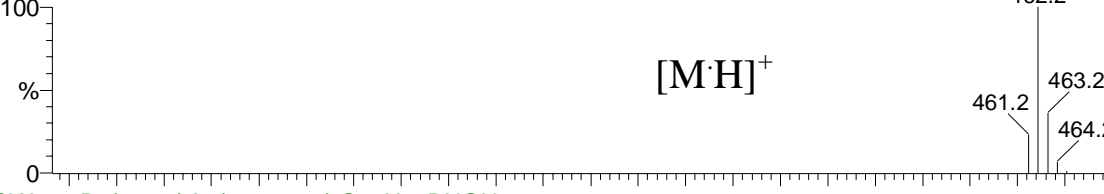

$\mathrm{M} \cdot \mathrm{H}]^{+}$

OF LD+

SKA058B $(0.051)$ Is $(1.00,0.10) \mathrm{C} 33 \mathrm{H} 24 \mathrm{BNONa}$

100


SKA058B 64 (3.195) Cm (2:71)

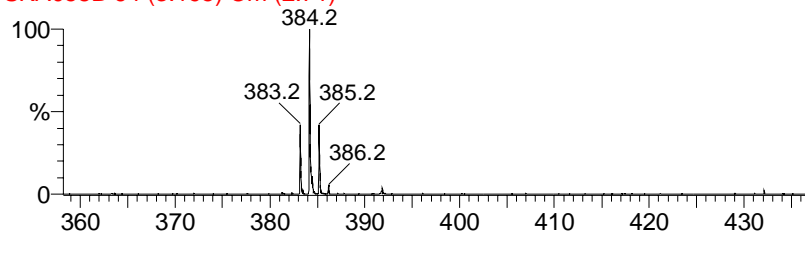

TOF LD

$41 \mathrm{e} 4$

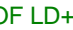

$02 \mathrm{e} 12$

SKA058B $(0.051)$ Is $(1.00,0.10)$ C33H24BNON

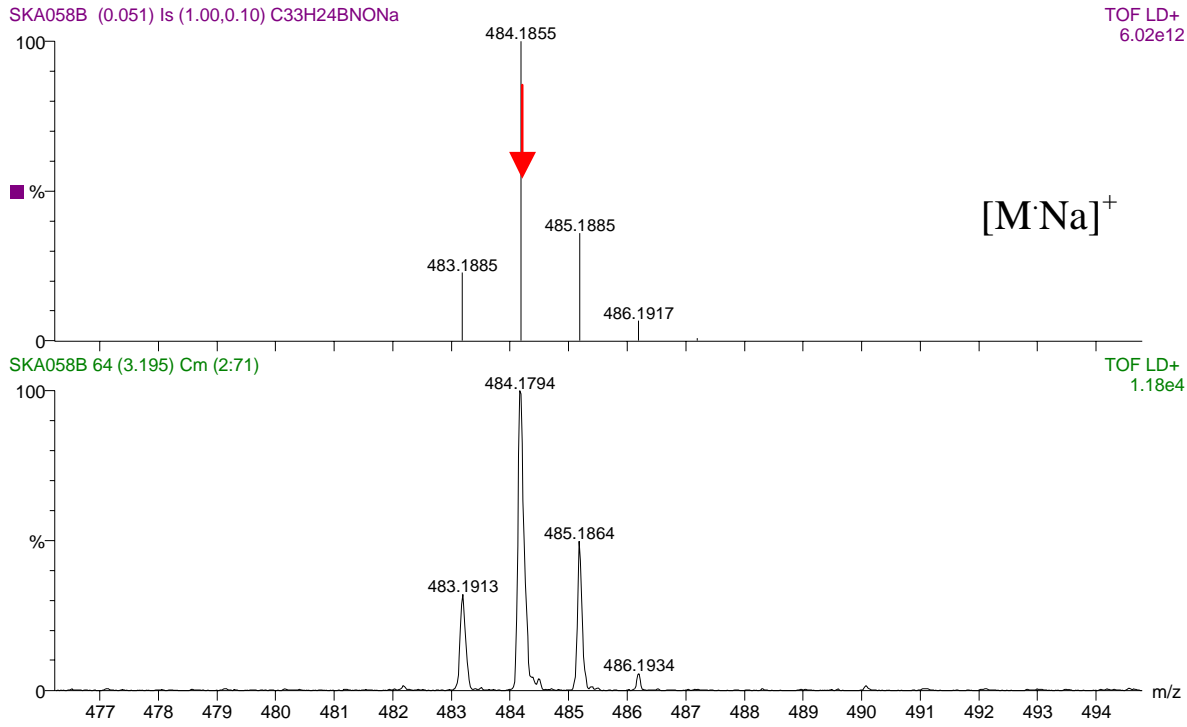


m rel.int.

$612.2613 \quad 0.2168$

$613.2585 \quad 1.0000$

$614.2614 \quad 0.4839$

$615.2646 \quad 0.1208$

$616.2678 \quad 0.0202$

$617.2710 \quad 0.0025$
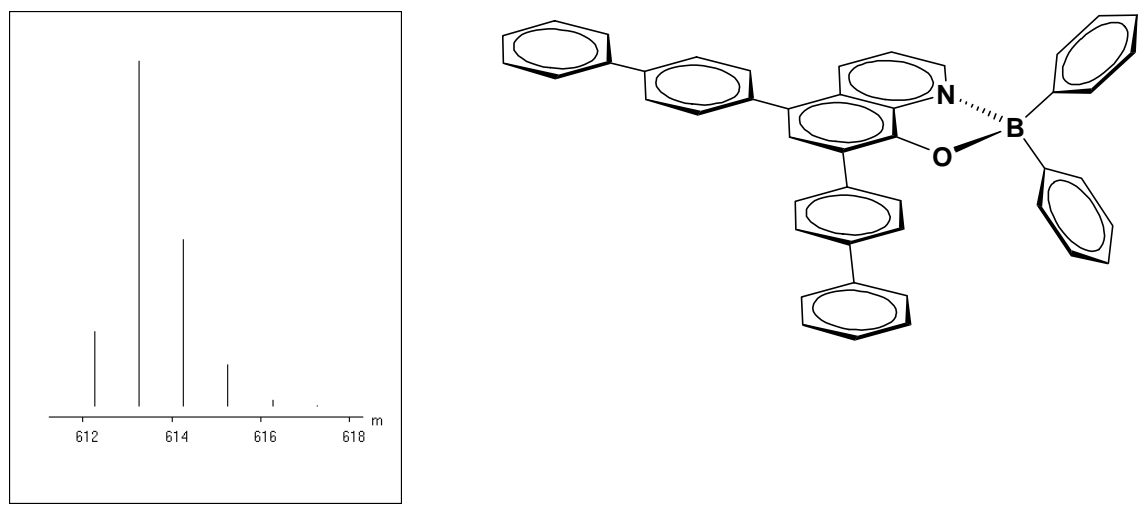

SKA066B (0.045) Is $(1.00,0.10) \mathrm{C} 39 \mathrm{H} 27 \mathrm{BNO}$

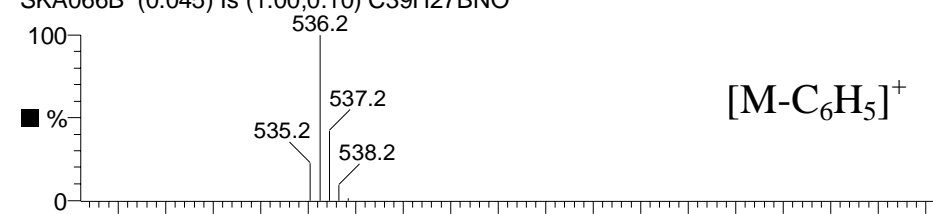

$\left[\mathrm{M}-\mathrm{C}_{6} \mathrm{H}_{5}\right]^{+}$ SKA066B $(0.045)$ Is $(1.00,0.10) \mathrm{C} 45 \mathrm{H} 32 \mathrm{BNOH}$
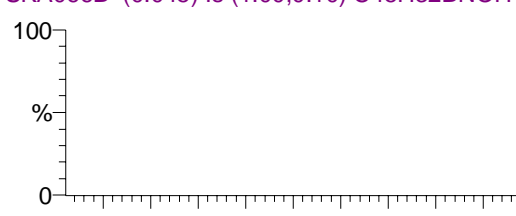

SKA066B $(0.045)$ Is $(1.00,0.10) \mathrm{C} 45 \mathrm{H} 32 \mathrm{BNONa}$

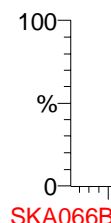

SKA066B 96 (4.885) Cm (17:100)
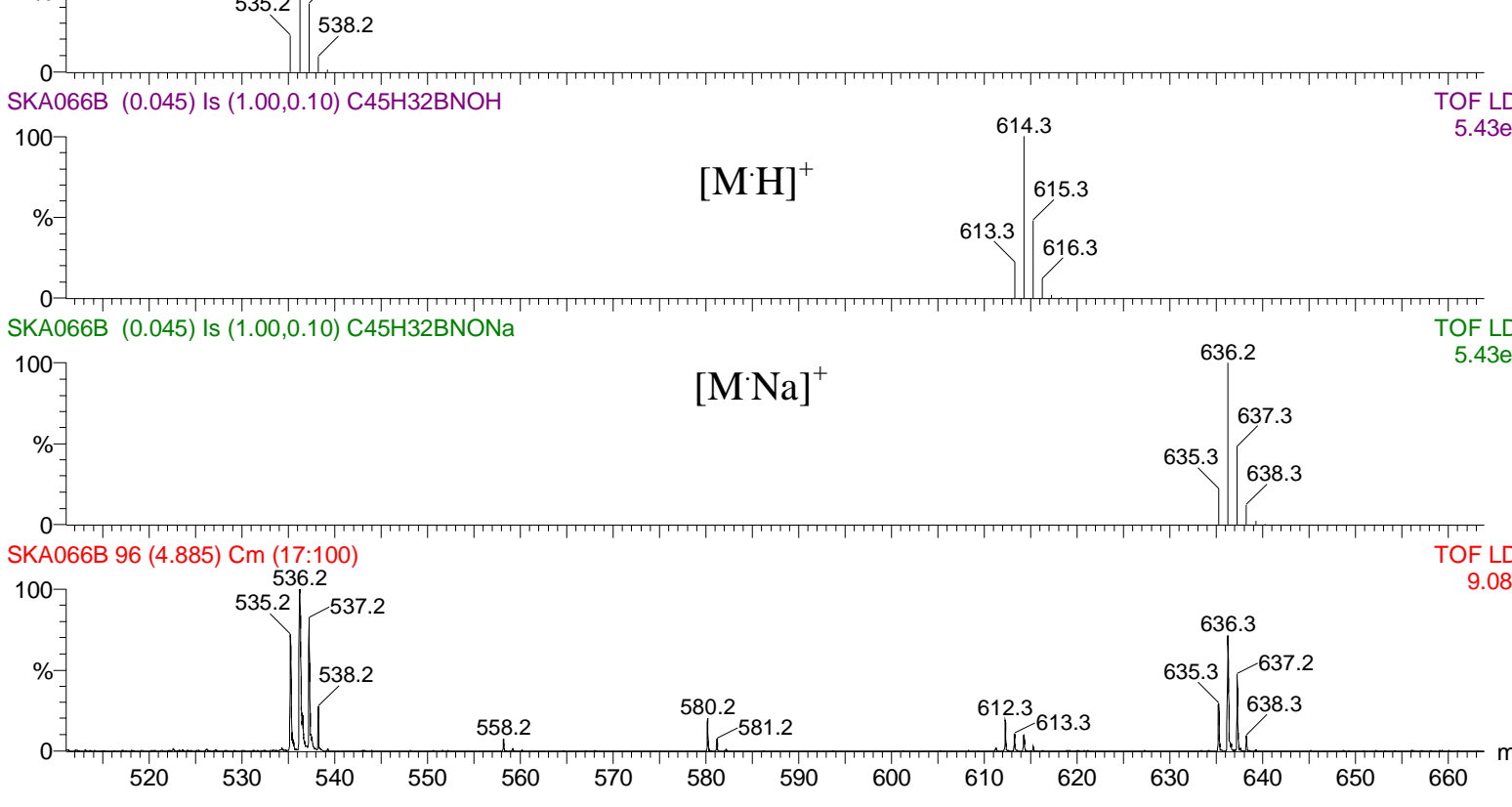
12 (=SKA069) $\quad \mathrm{C}_{71} \mathrm{H}_{80} \mathrm{BNO} \quad \mathrm{M}_{\mathrm{av}}=974.2466$

m rel.int.

$972.6369 \quad 0.2037$

$973.6345 \quad 1.0000$

$974.6373 \quad 0.7433$

$975.6405 \quad 0.2901$

$976.6437 \quad 0.0761$

$977.6470 \quad 0.0149$

$\begin{array}{ll}978.6502 & 0.0023\end{array}$
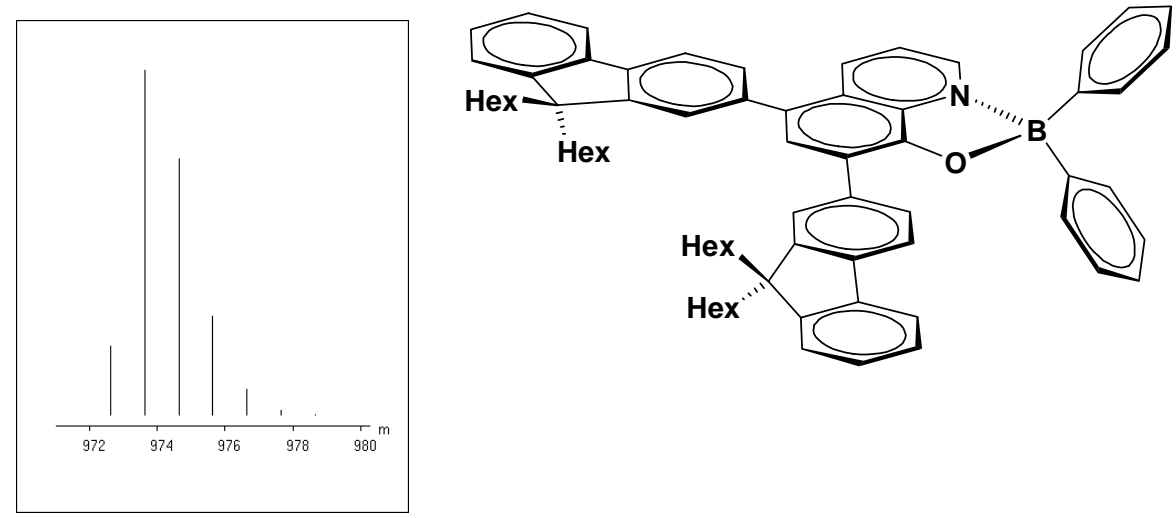

SKA069B (0.042) Is $(1.00,0.10) \mathrm{C} 65 \mathrm{H} 75 \mathrm{BNO}$



TOF LD+

$4.54 \mathrm{e} 12$

SKA069B $(0.042)$ Is $(1.00,0.10) \mathrm{C} 71 \mathrm{H} 80 \mathrm{BNOH}$
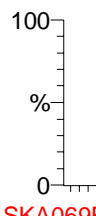

SKA069B $(0.042)$ Is $(1.00,0.10)$ C71H80BNONa
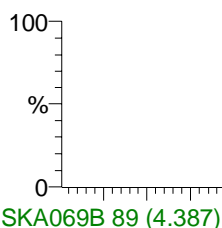

(4.387) Cm (17:92)

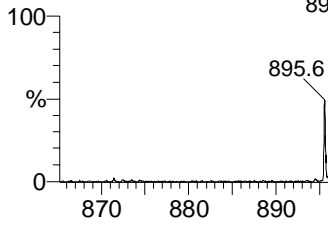

897.

$\left[\mathrm{M}-\mathrm{C}_{6} \mathrm{H}_{5}\right]^{+}$

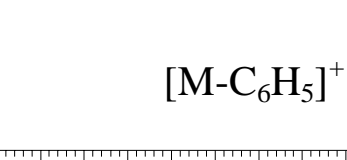




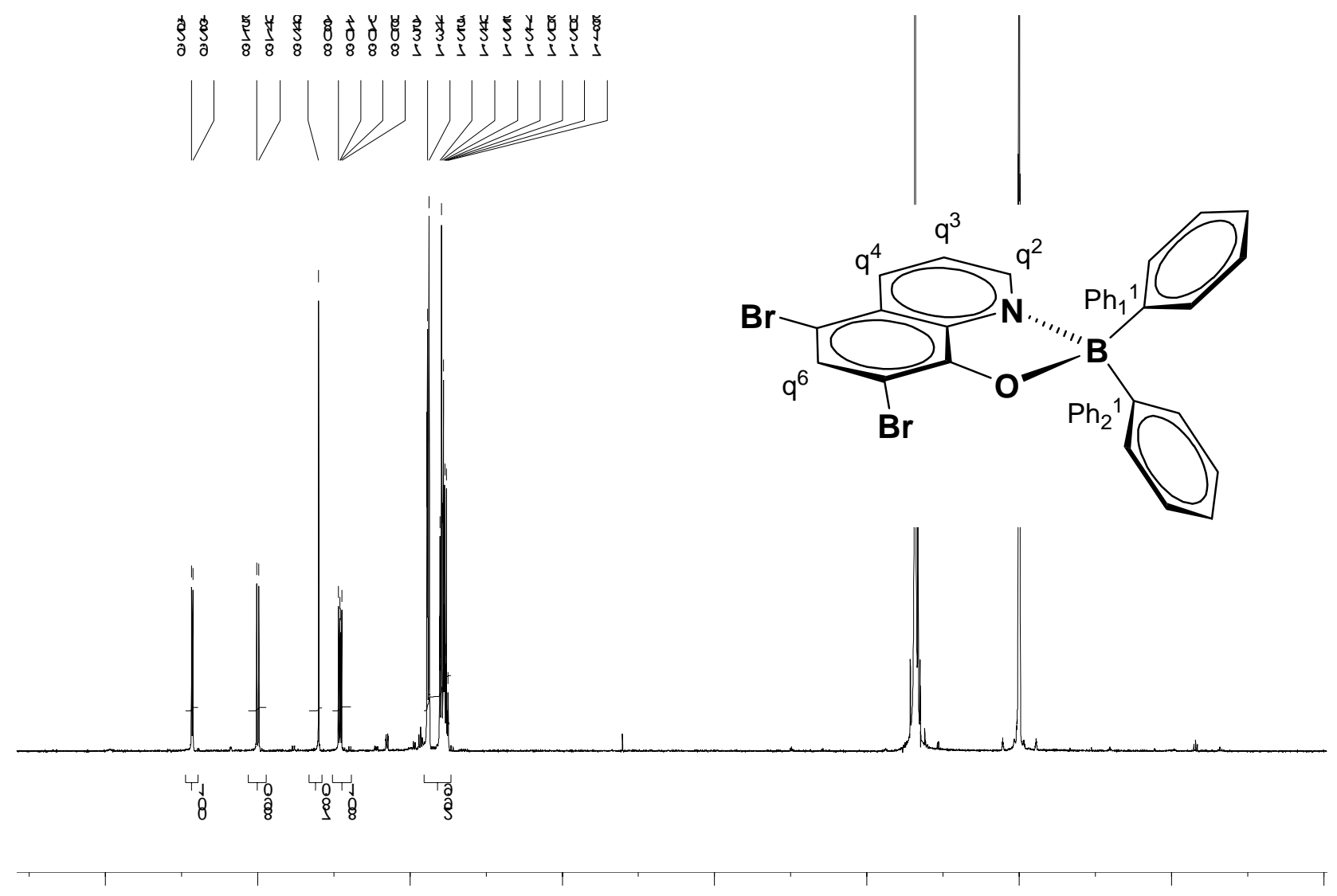

HhH"y
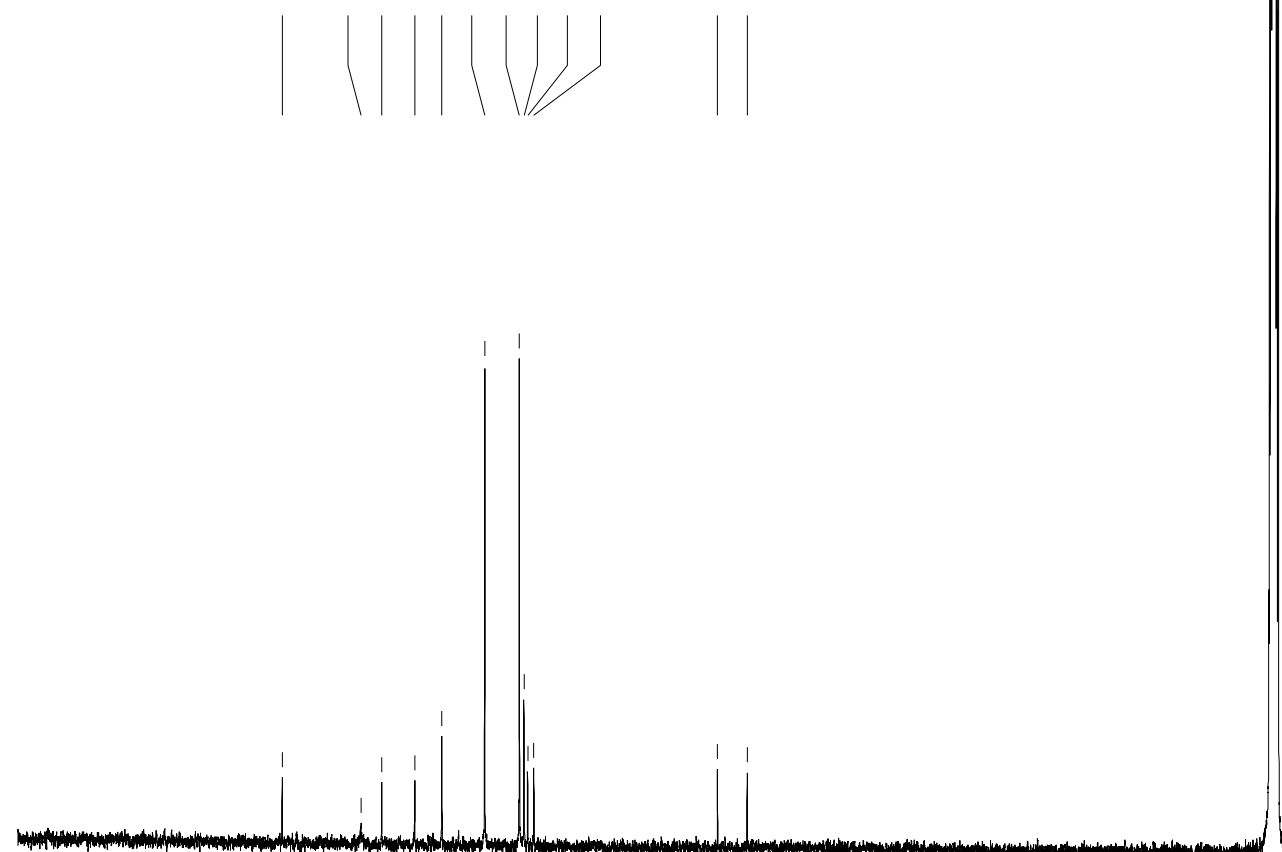


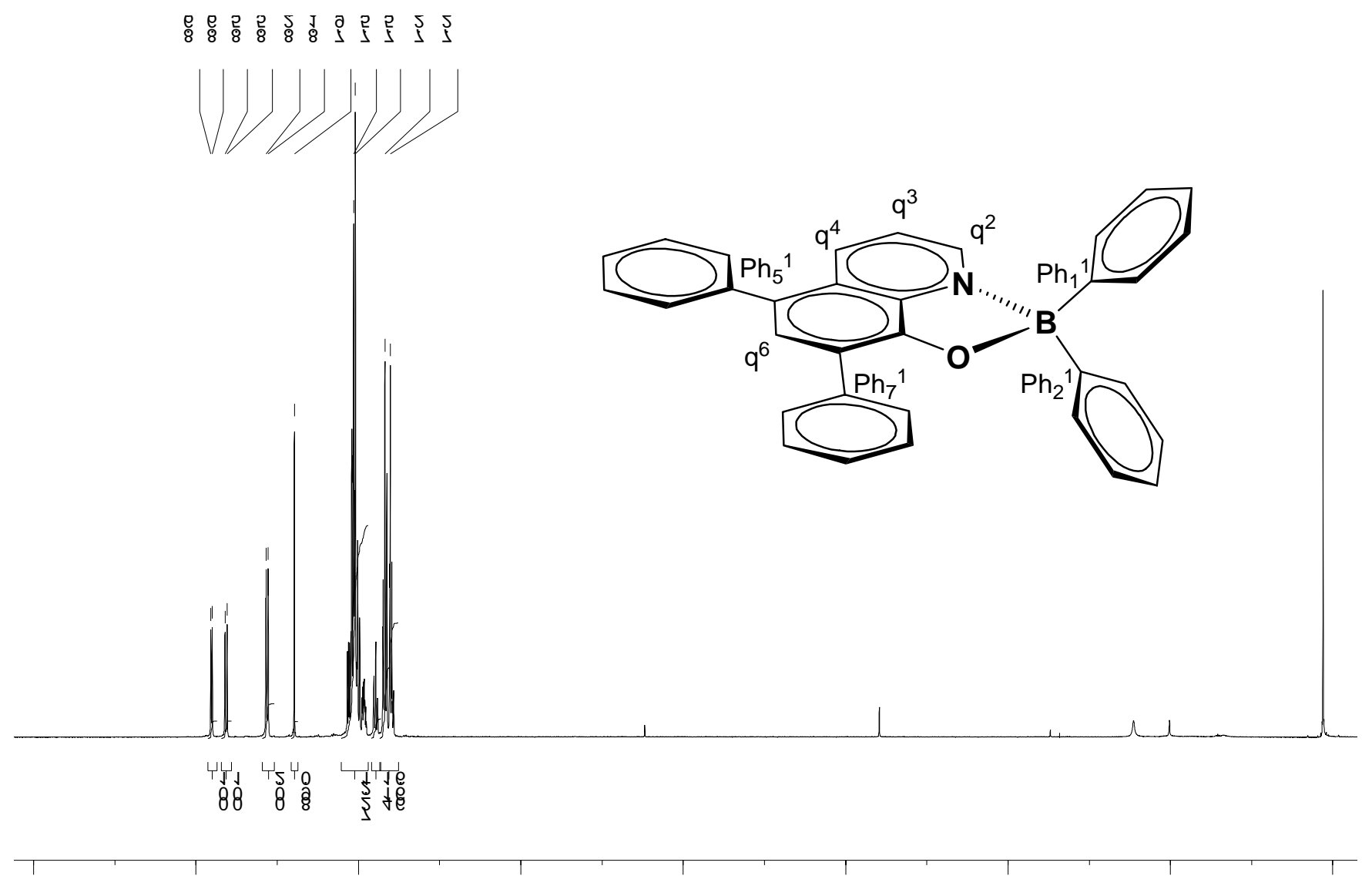


ImmInmm!n!

curculyenty

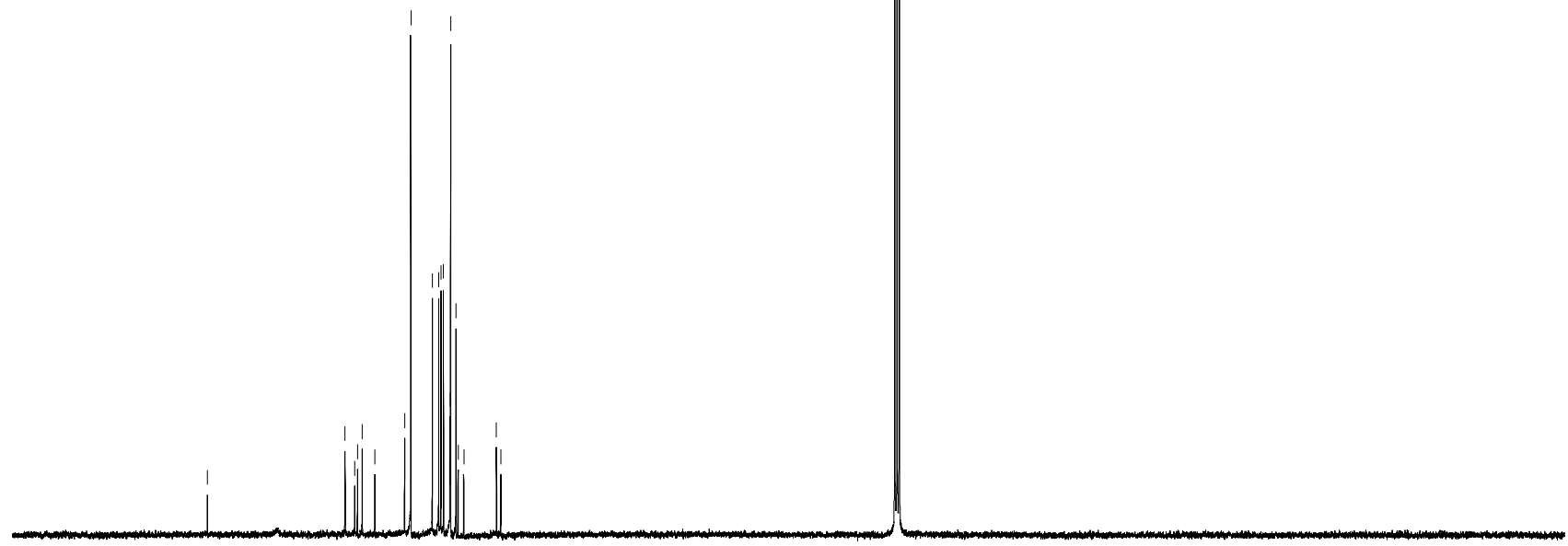

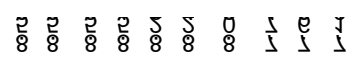

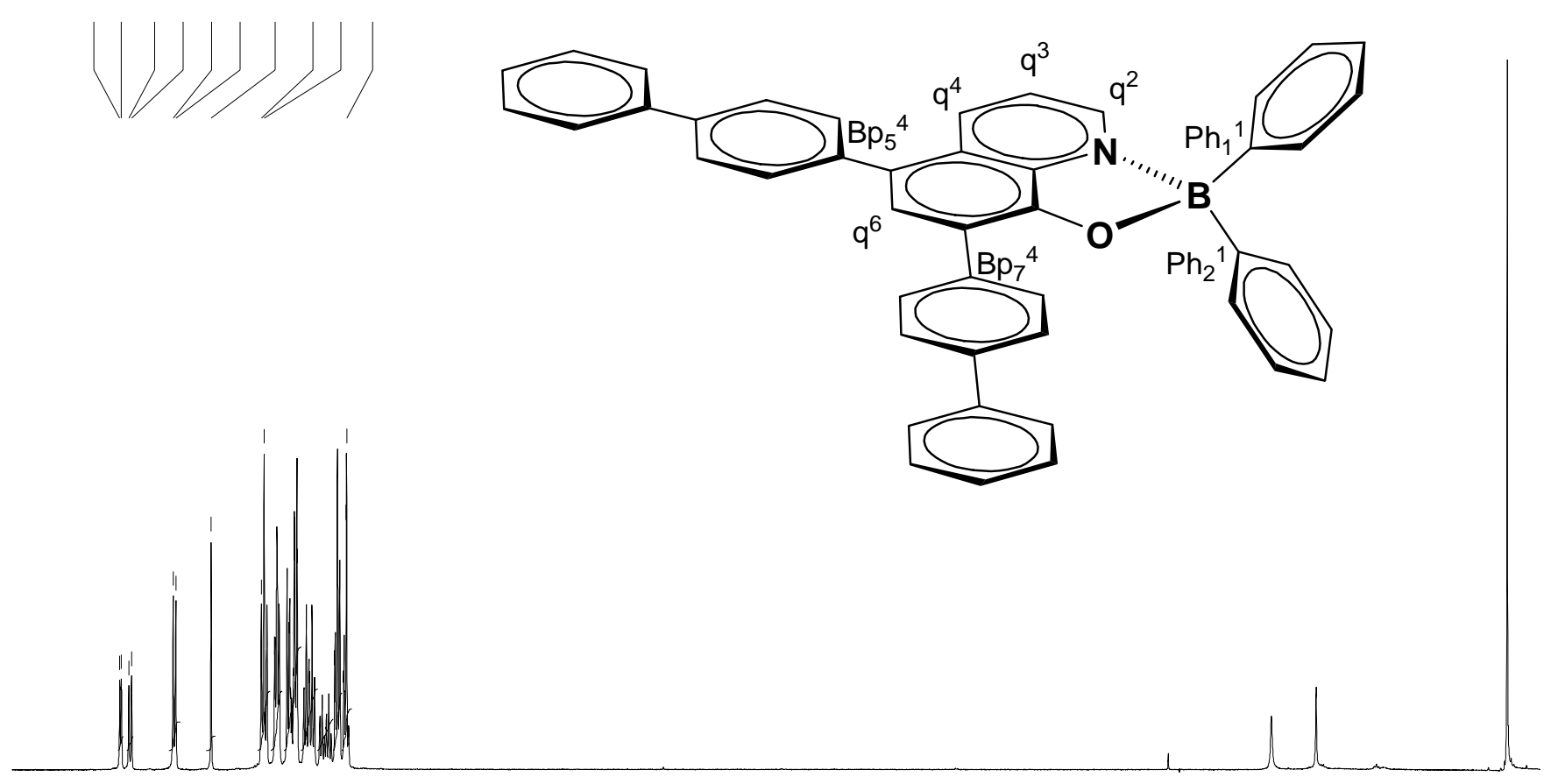

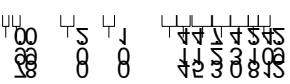




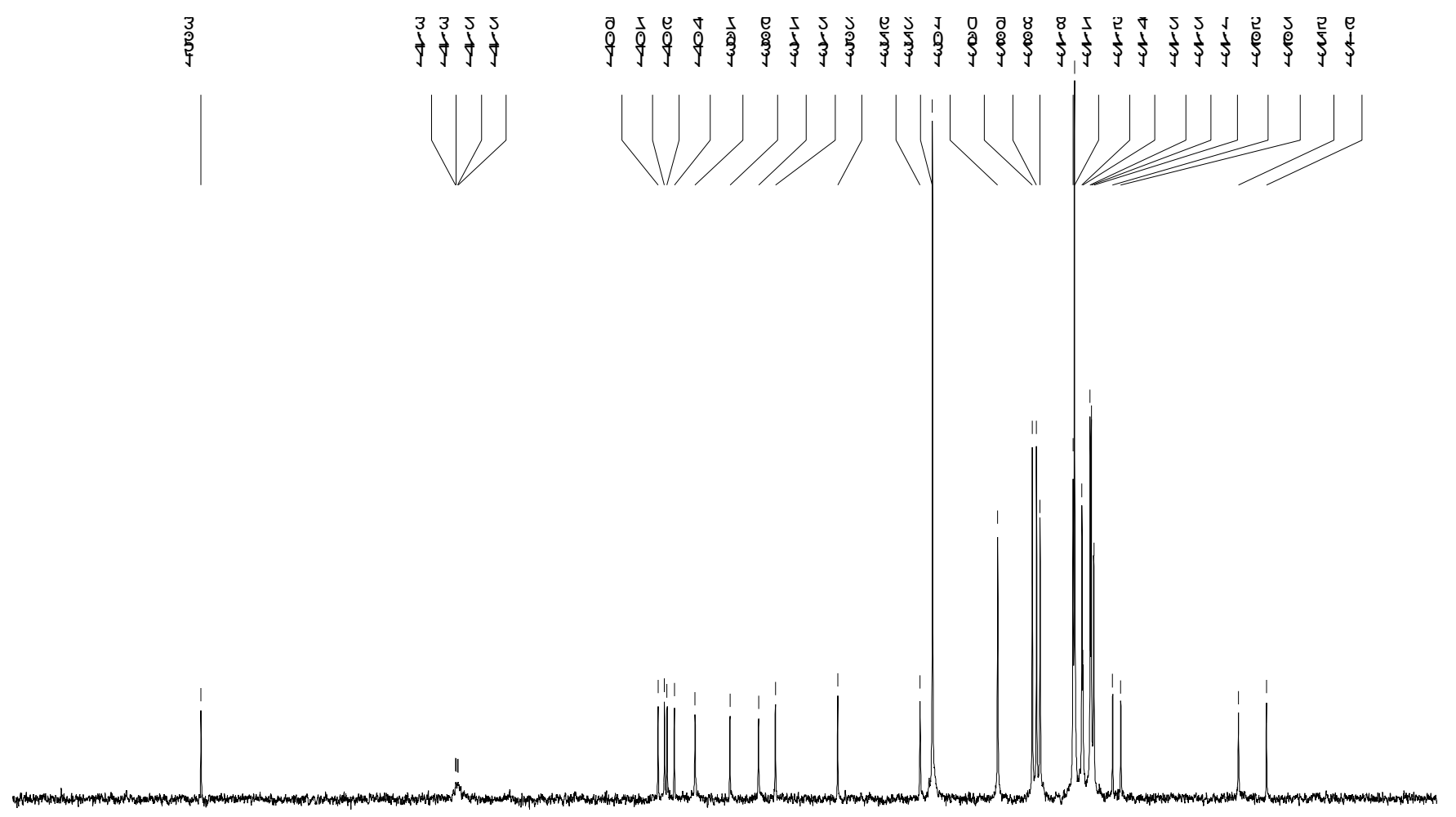




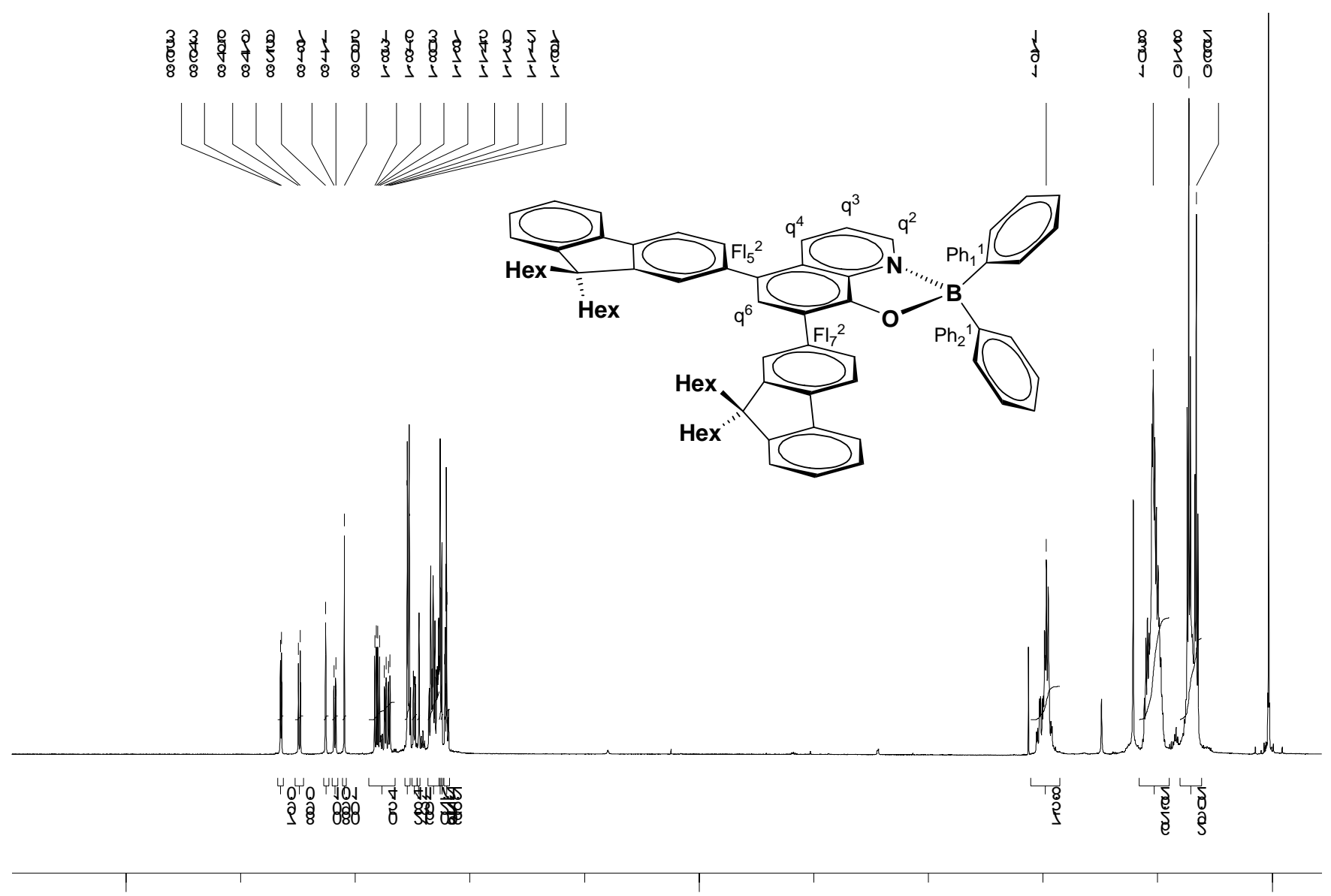



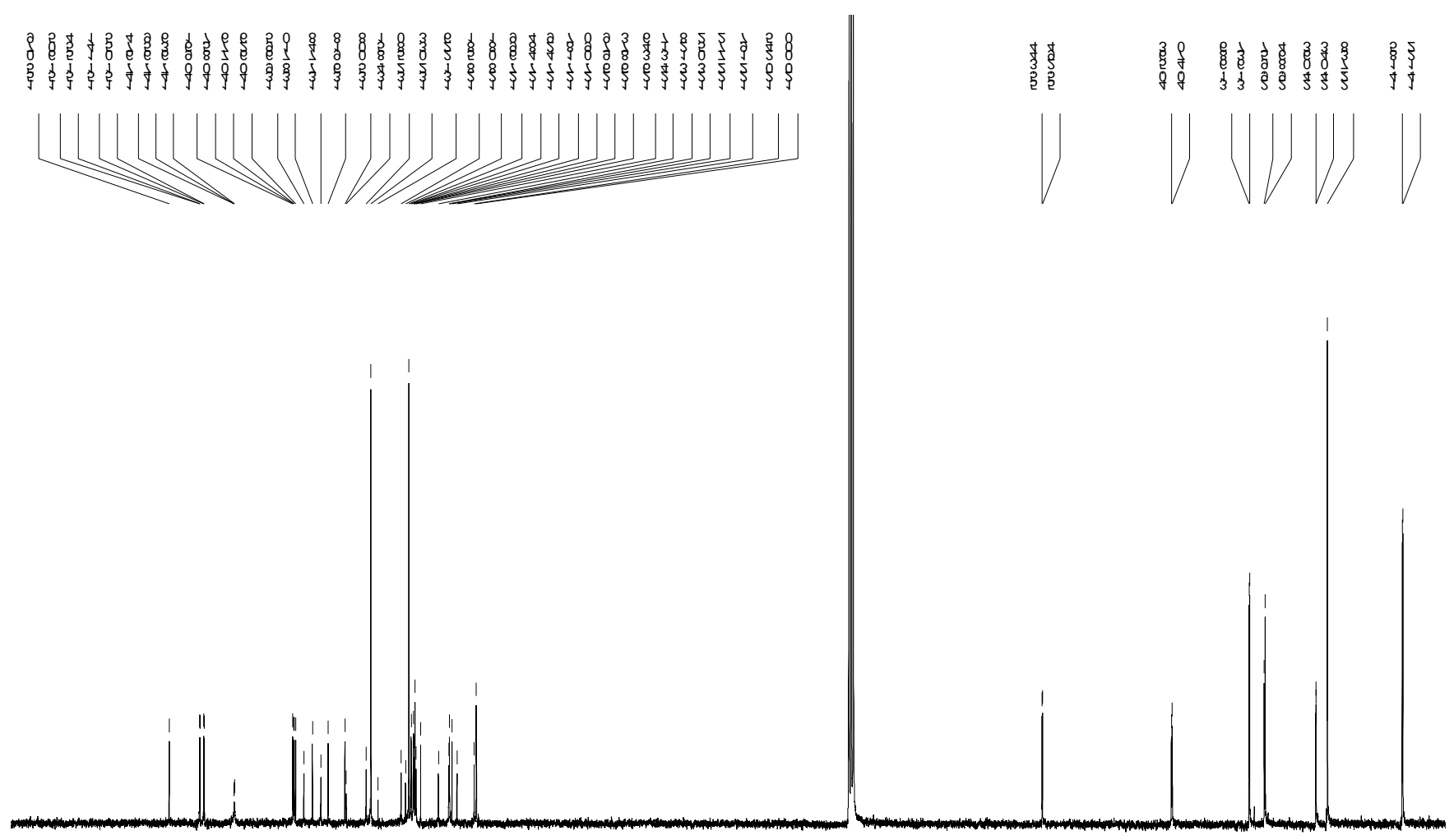

Photoluminescence of films of compounds 9 - 12 

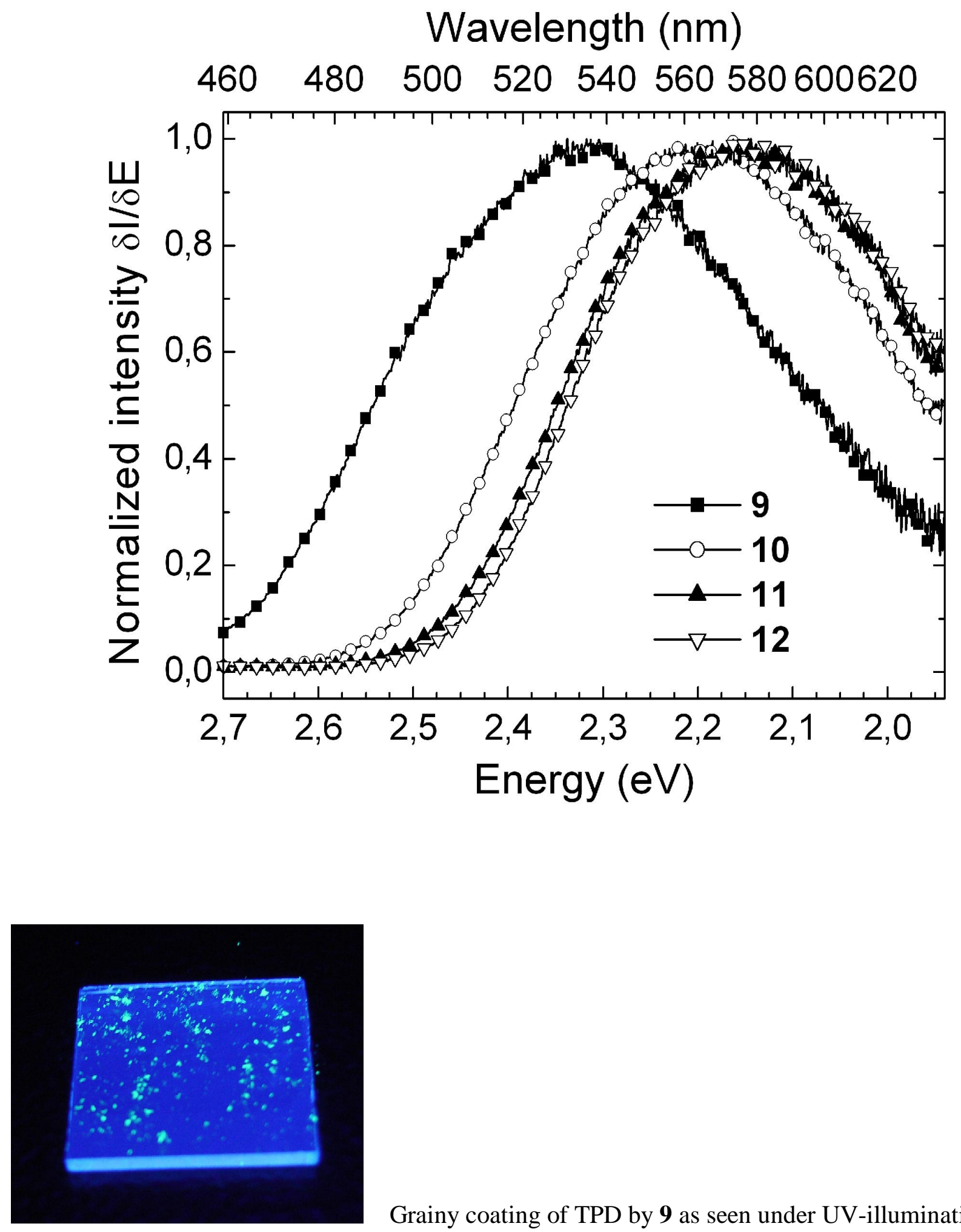

Grainy coating of TPD by $\mathbf{9}$ as seen under UV-illumination 


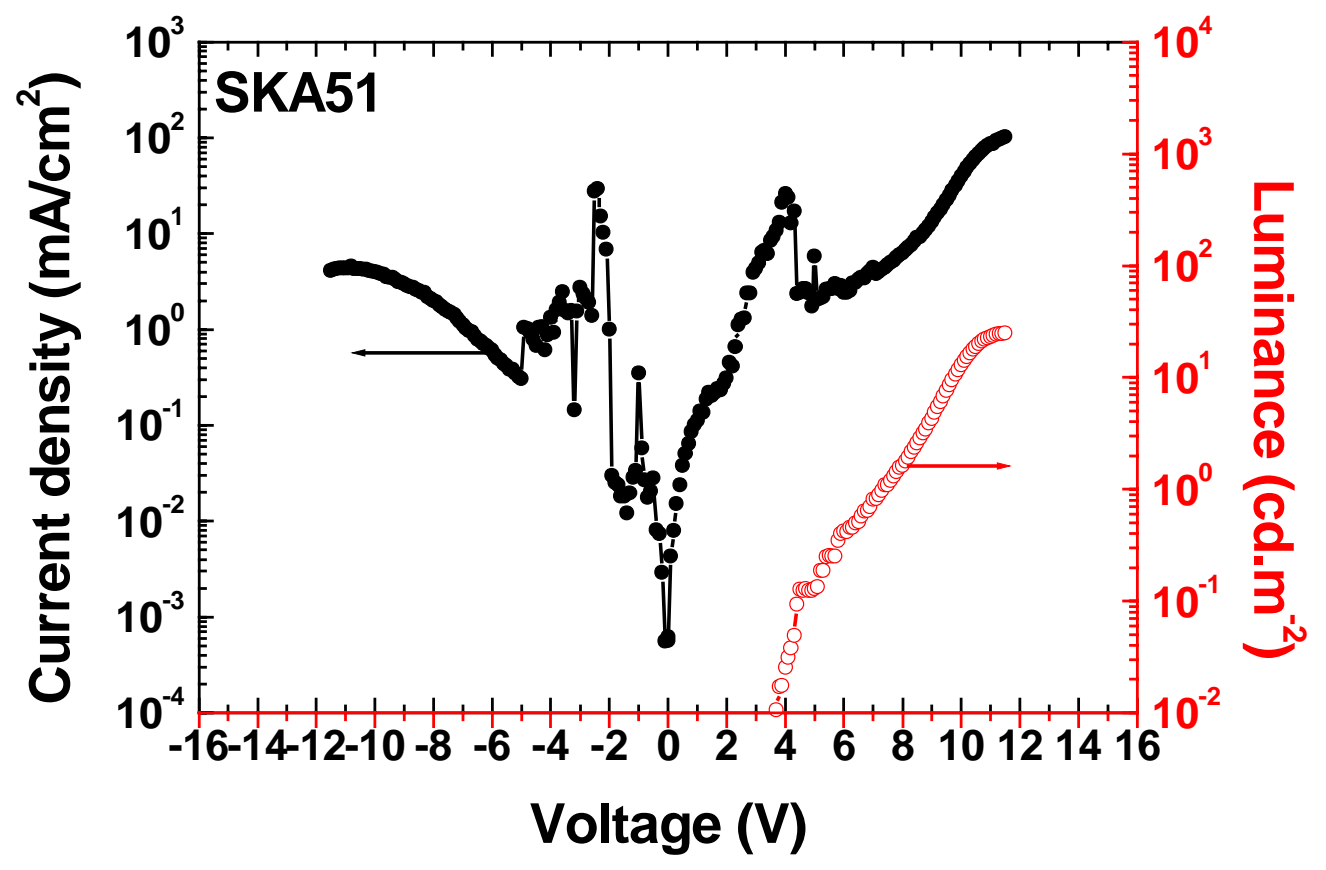

Current density - voltage characteristics of a device made from 9 (= SKA51).



Current density - voltage characteristics of a device made from 10 (= SKA58). 


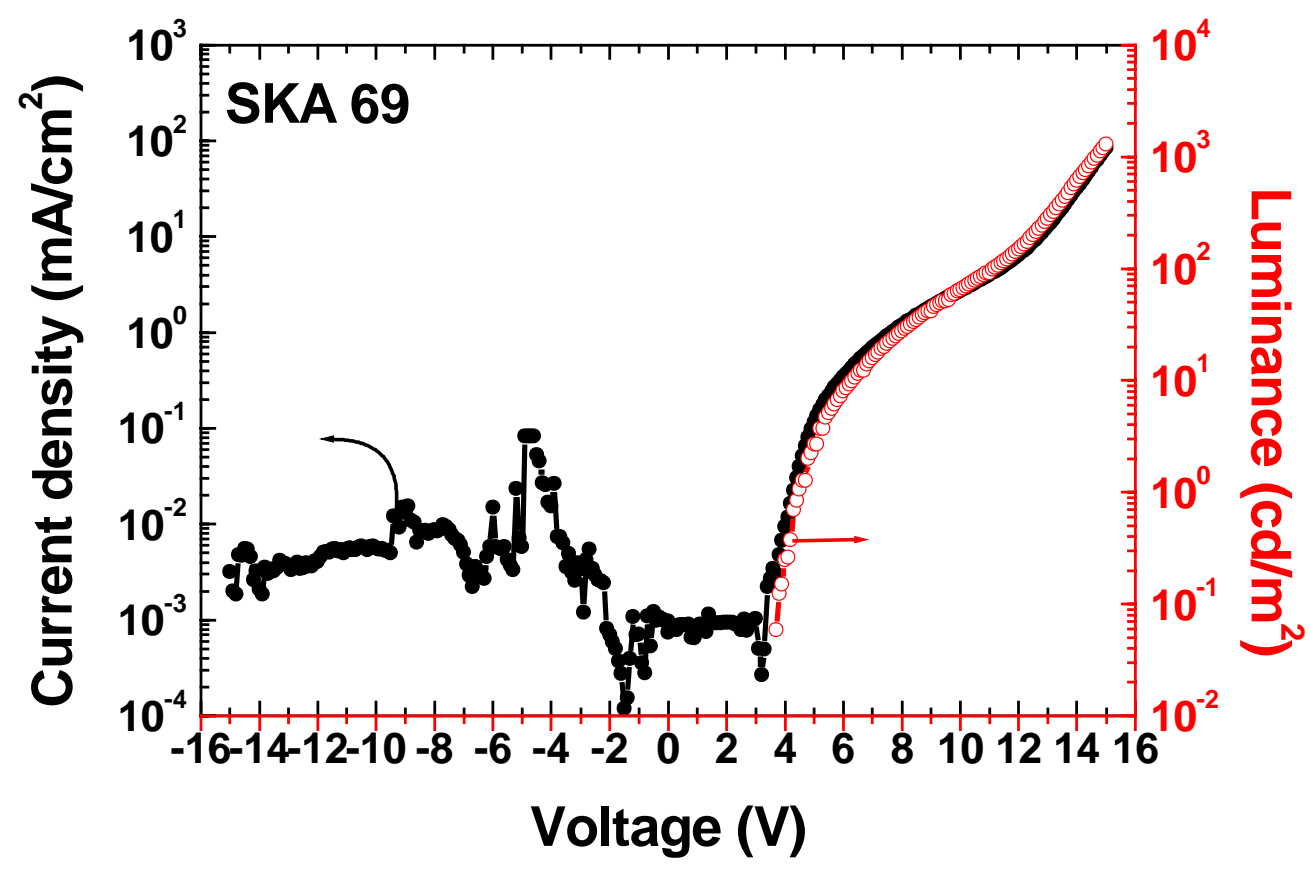

Current density - voltage characteristics of a device made from 12 (= SKA69). 